\title{
6 Umweltschutz, \\ Klimawandel und \\ kritischer Konsum
}

Dieses Kapitel wurde bei Erstveröffentlichung ohne die korrekte Creative

Commons Lizenz veröffentlicht. Die korrekte Lizenz finden Sie am Ende des Kapitels.

Ein Erratum zu diesem Kapitel ist verfügbar unter DOI 10.1007/978-3-658-12533-2_14

(C) SINUS Markt- und Sozialforschung GmbH 2016

M. Calmbach et al., Wie ticken Jugendliche 2016?,

DOI 10.1007/978-3-658-12533-2_6 
Unsere Gesellschaft steht mit Blick sowohl auf ökologische und ökonomische als auch auf soziale Entwicklungen vor diversen Herausforderungen: Energiewende, Klimafolgenanpassung, globale Armutsbekämpfung oder das Aufhalten der fortschreitenden Verschmutzung der Weltmeere sind nur wenige Schlagworte in diesem Kontext. Aufgrund der Vielschichtigkeit und globalen Komplexität dieser Aufgaben erscheinen Lösungen umso schwieriger - nicht nur in der Entwicklung, sondern auch in der Vermittlung und Durchführung in globalen Zusammenhängen.

In diesem Kapitel werden ausgewählte Themenfelder im Kontext dieser globalen Herausforderungen aus der lebensweltlichen Perspektive der Jugendlichen beleuchtet - nämlich Umweltschutz, Klimawandel und „kritischer Konsum". Wir möchten erfahren, wie es in der jungen Generation um die Bereitschaft zur sozialen und ökologischen Verantwortungsübernahme bestellt ist und wie sie ihre Handlungswirksamkeit hierbei einschätzt. Die Themen Umweltschutz und Klimawandel sind medial regelmäßig präsent. Beispielsweise sind Weltklimakonferenzen (z. B. in Paris) und die Diskussion um die Energiewende Top-Nachrichtenthemen. Was davon aber kommt bei den Jugendlichen an und welche Meinung haben sie dazu? Inwieweit möchten sie sich für Umwelt- und Klimaschutz engagieren und was hindert sie daran?

Mit großer Regelmäßigkeit wird in den Medien zudem über Lebensmittel- und Tierschutzskandale, Produktionsbedingungen in Schwellenländern oder auch den Trend zum Bio- und Slow-Food berichtet. Neben Umweltschutz und Klimawandel wurde daher auch „kritischer Konsum" als weiteres Schwerpunktthema im Kontext ökologischer und sozialer Verantwortungsübernahme ausgewählt. Gemeint ist mit diesem Begriff, dass Konsumentinnen und Konsumenten ihre Kaufentscheidungen davon abhängig machen, wo das Produkt herkommt, unter welchen Bedingungen es hergestellt wurde und welche Transportwege es hinter sich hat.

Konsum spielt in der Identitätsentwicklung junger Menschen eine zentrale Rolle, da sich über Kleidung, Smartphones und andere Konsumgegenstände Zugehörigkeit und Abgrenzung in jugendlichen Lebenswelten herstellen lassen. Große, günstige Modeketten haben die jungen Zielgruppen fest im Blick. Das Gleiche gilt für die bekannten 
Fast-Food-Anbieter. Insofern ist die Frage, ob Jugendliche beim Konsum auch Kriterien der Herstellung berücksichtigen wollen und können, besonders interessant. Die Art und Weise, wie man sich über Produkte informiert und die Frage, was, wie und warum konsumiert wird, bündelt zudem soziale, ethische, ökologische und ökonomische Facetten der Verantwortungsübernahme. Von Interesse ist daher auch, welche Dimensionen für Jugendliche am ehesten entscheidungs- und damit handlungsrelevant sind.

\subsection{Umweltschutz}

\section{Umweltschutz ist aus Sicht der Jugendlichen eine der großen Herausforderungen für die Zukunft, an der die Menschheit vielleicht sogar scheitern wird.}

Das Thema Umweltschutz gehört für fast alle Jugendlichen zu den zentralen gegenwärtigen und zukünftigen Herausforderungen. Dabei wird das Thema häufig spontan genannt und emotional vorgetragen. Die Jugendlichen sehen klar die Menschen selbst in der Verantwortung bzw. in der Schuld für die gegenwärtigen drastischen Umweltprobleme.

Die globalen und langfristigen Zusammenhänge haben dabei eher die Sozialökologischen und Expeditiven Jugendlichen sowie teilweise auch die Experimentalistischen Hedonisten im Blick. Beispiele und Themen aus dem eigenen (lokalen) Alltag (z.B. Mülltrennung, Energiesparen) werden häufiger von den Konservativ-Bürgerlichen und Materialistischen Hedonisten genannt.

Jugendliche halten das Thema Umweltschutz zwar für außerordentlich wichtig (vereinzelt auch mit Blick auf intergenerationelle Gerechtigkeit), gleichzeitig haben sie nur wenig Vertrauen, dass diese Aufgabe auch tatsächlich bewältigt werden kann. Man zeigt sich sehr besorgt, dass sich die Zerstörung des Planeten nicht aufhalten lässt. 
Typische Zitate zur |llustration
Ja, dass man halt verantwortungsvoll mit seiner Umwelt umgeht und auch daran denkt, dass die Leute in 70 Jahren auch noch auf der Welt sind, und nicht nur an seine eigene Lebenszeit denkt. (weiblich, 17 Jahre, Sozialökologische)

Also eine Herausforderung ist eigentlich nur, dass man nach der Umwelt schauen muss, weil, ja, natürlich Erderwärmung und alles. Und dass die Polare schmelzen und alles. Eigentlich ist das größte Thema der Planet an sich. (weiblich, 15 Jahre, Experimentalistische Hedonisten)

(8) Die Verschmutzung im Wald und im Meer, das ist ganz schlimm. Ich meine, wir sind auf der Welt, aber das ist ja nicht unsere Welt eigentlich. Wir werden ja hier nur ertragen quasi. Und dann können wir uns das ja nicht erlauben, die einfach kaputt zu machen. (weiblich, 17 Jahre, Konservativ-Bürgerliche)

( Ja, Umwelt ist mir sehr wichtig. Weil wir müssen in der Umwelt auch leben. Wenn man jetzt die Luft verpestet, dann fragen sie sich, warum es keine saubere Luft mehr gibt. Und eigentlich sind sie selber daran schuld. (weiblich, 17 Jahre, Materialistische Hedonisten)

Wir haben nur einen Planeten. Und wenn wir den zugrunde richten, sehen wir alt aus. Und deshalb sollten wir da schon ein bisschen gucken, was wir daraus machen. (männlich, 15 Jahre, Sozialökologische)

8 Eigentlich finde ich das ziemlich gut, weil es ist ja sehr wichtig, die Umwelt zu schützen. Weil wenn die kaputt gemacht wird, wird ja auch unser Lebensraum kaputt gemacht. (weiblich, 15 Jahre, Sozialökologische)

( Aber es gibt ja immer noch die ganzen großen Probleme, die sich nicht einfach in Luft auflösen. Letztendlich zerstört der Mensch ja auch den ganzen Planeten. Und ich weiß nicht, ob sich das ändern wird. Ich glaube nicht. (weiblich, 16 Jahre, Sozialökologische)

( Kein Tier würde seinen eigenen Planeten kaputt machen. Keines! Was macht aber der Mensch: Scheiß drauf! Warum? Wahrscheinlich brauchen wir irgendwann einen neuen Planeten, weil wir so doof waren und alles zerstört haben. Wir haben alle Wälder abgeholzt. Wir haben alle Tiere getötet. (weiblich, 16 Jahre, Experimentalistische Hedonisten) 


\section{Umweltschutz wird als wichtig anerkannt - vor allem, wenn es um die Gefährdung der eigenen Lebensqualität geht.}

Den meisten Jugendlichen fallen beim Stichwort Umweltschutz sofort mehrere konkrete Themen ein - unabhängig davon, über welches Bildungsniveau sie verfügen und welcher Lebenswelt sie angehören. Auch Klimaschutz und nachhaltiger Konsum werden von den Jugendlichen hier zugerechnet.

Spontan genannt werden u. a. die Müllproblematik, der Klimawandel, die Abholzung des Regenwaldes, die Luftverschmutzung, Natur- und Tierschutz, die Atomenergie und alternative Energien, vegetarische und vegane Ernährung bzw. Fleischkonsum, der öffentlicher Nahverkehr und Autoverkehr, der Natur- und Tierschutz, das Konsumverhalten und Recycling.

Vorrangig werden bei Umweltschutz nicht die Folgen für Menschen und Natur in globaler Perspektive beschrieben, sondern das Thema auf den eigenen (Nutzungs-)Kontext von Umwelt heruntergebrochen. Umweltschutz wird für Jugendliche v. a. relevant bzw. erleb- und sichtbar, wenn sie Einschränkungen ihres persönlichen Erlebnisraumes befürchten, z. B. bestimmte Tiere nicht mehr sehen oder essen zu können.

(8) Mir geht da so die Ozonschicht durch den Kopf, die die Erde umgibt. Weil ich halt Angst habe, weil ja so viel Müll produziert wird und Plastik und so, dass es einfach irgendwann einmal zerplatzt oder was auch immer. Und es ist schon echt grausam, wenn man mal sieht, wie viel Plastik man eigentlich verbraucht und generell Müll und Lebensmittel wegschmeißt. Das ist halt blöd. (weiblich, 14 Jahre, Konservativ-Bürgerliche)

Ich liebe Wale, früher war ich so voll der Wal-Typ. Jetzt nicht mehr so, aber ich finde, dass man Wale fängt und Walfleisch ißt, gar nicht gut. Auch so etwas wie Regenwald abholzen finde ich alles gar nicht gut. (männlich, 15 Jahre, Materialistische Hedonisten) 
Unter Umweltschutz verstehe ich viel, alles: Atomkraftwerke abschalten. Mehr auf die Umwelt achten, weniger Abgase produzieren. Weniger mit dem Auto fahren, das ist ja ungefähr das Gleiche. Umweltfreundliche Verkehrsmittel nutzen zum Beispiel, wie das Fahrrad. Ja, neue Techniken, die es halt ermöglichen, umweltfreundlicher zu leben. (männlich, 17 Jahre, Experimentalistische Hedonisten)

( Das ist dann so etwas wie vegetarisch sein, dass man besonders darauf achtet, dass es einen geringen CO2-Ausstoß hat. (männlich, 17 Jahre, Konservativ-Bürgerliche)

Umwelt sollte auf jeden Fall auch geschützt werden, vor allem auch die Tiere. Neulich ist es ja wieder vorgekommen, dass ein Deutscher ja auch den größten Elefanten getötet haben soll. Dass einfach die Tiere und die Umwelt geschützt werden, weil wir rotten die Wälder aus. Ich glaube auch, dass in der Zukunft überall künstliche Luft ist. Dass es vielleicht sogar so ist, dass man zu einer Mikrowelle geht oder zu irgendwas, zu einer Box, und sich dann sprachgesteuert bedient und alles per Chemie in der Mikrowelle ist. Dass man dann auch nicht mehr selber kocht, sondern dass alles nur chemisch ist. Auf die Umwelt sollte man daher definitiv mehr Wert legen. (weiblich, 14 Jahre, Hauptschule, Experimentalistische Hedonisten)

( Umweltschutz? Ich denke da immer so an die Tiere, weil Naturschutz so. Manche stehen dann so unter Naturschutz, zum Beispiel gibt es nur noch einen schwarzen Löwen, den wollte ich auch mal sehen, so live so eigentlich, aber den findet man ja irgendwo nicht so wirklich. Da denke ich schon eher dran, als jetzt zum Beispiel Abgase. Das ist ja auch so ein großes Thema (männlich, 15 Jahre, Prekäre)

(7hunfisch soll ja auch aussterben. Was mich enttäuscht, weil ich mag Thunfisch. Thunfisch ist lecker, mit Mais, Salat, Joghurtdressing. (männlich, 15 Jahre, Konservativ-Bürgerliche)

Wenn Jugendliche sich selbst als schlecht informiert beschreiben oder an den wissenschaftlichen Beweisen zweifeln (z. B. beim Thema Klimawandel), messen sie dem Thema Umweltschutz eine entsprechend geringere Bedeutung zu. Vor allem männliche und eher konservative Jugendliche sind skeptisch beim Thema Umweltschutz. Sie erwähnen 
- zumeist unspezifisch - dass viel Geld für Umweltmaßnahmen (z.B. Förderung erneuerbarer Energien) ausgegeben wird, wobei nicht immer klar sei, ob dies tatsächlich etwas bringen würde.

Umweltschutz ist so eine Sache. Ich bin zwar dafür, aber was wir dort an Kosten reinstecken, ist einfach nicht effizient. (männlich, 17 Jahre, Konservativ-Bürgerliche)

\section{Informationen zum Umweltschutz beziehen Jugendliche vor allem aus der Schule und aus den Medien.}

Ihre Informationen zum Thema Umweltschutz haben die Jugendlichen vor allem aus der Schule. Teilweise äußern die Jugendlichen sogar den Wunsch, dem Thema im Unterricht mehr Raum zu geben. Vereinzelt sind Beiträge aus dem Fernsehen oder Internet in Erinnerung (alarmierende Dokumentationen, Foren zu speziellen Themen). Daneben sind auch Nachrichten mögliche Quellen, aus denen man - mehr oder weniger zufällig - etwas über Umweltschutz erfährt. Die aktive eigene Informationssuche und Recherche zum Thema wird nur vereinzelt erwähnt.

In der Familie und im Freundeskreis ist Umweltschutz eher in den Lebenswelten in höherer Bildungslage ein Gesprächsthema.

(7) Wir haben in der Schule im Geografieunterricht viel über Ressourcen geredet, also über Erdöl und Erdgas und auch Wasser. Und auch über die entsprechenden Prognosen. (weiblich, 17 Jahre, Adaptiv-Pragmatische)

( Wir reden in der Schule auch viel über den Klimawandel, auch in anderen Fächern wie Englisch. (weiblich, 17 Jahre, Adaptiv-Pragmatische)

( Ich habe mir da mal so eine Doku angesehen auf n24, glaube ich. Aber ich kann mir zum Beispiel Dokus über Klimawandel oder so nicht angucken, weil ich das immer so schrecklich finde, wenn man so was sieht. Auch dieser ganze Plastikmüll. (männlich, 14 Jahre, Materialistische Hedonisten) 


\section{Umweltschutz im Alltag ist für die Jugendlichen eine selbstverständliche, dennoch nicht einfach durchzuhaltende Praxis.}

Die Jugendlichen sehen sich persönlich verantwortlich für den Umweltschutz. Immer wieder verweisen sie darauf, dass man mit kleinen Schritten im Alltag schon viel tun kann. Dieser Umweltschutz ist für Jugendliche mit konkreten Aktivitäten bzw. Handlungsoptionen verbunden. Im Vordergrund stehen dabei Aspekte der Müllvermeidung und des Recyclings (Müll trennen, Plastikflaschen recyclen), aber auch Maßnahmen zur Ressourcenschonung werden genannt, z. B. weniger Auto fahren oder "duschen statt baden". Umweltschutz wird somit vorrangig mit der Erhaltung einer sauberen, d. h. aufgeräumten Umwelt assoziiert: Dass man seinen Müll nicht auf die Straße wirft, wird bereits als aktiver Umweltschutz gewertet.

Zugleich thematisieren Jugendliche ihre eigene Nachlässigkeit. Insbesondere mit Blick auf Müllvermeidung und -trennung gesteht man sich ein, die selbst gesetzten Spielregeln immer wieder zu verletzen. Die eigene Vergesslichkeit oder Bequemlichkeit stehen hier dem konsequenten Umweltschutz im Weg.

Auch Sozialökologische und Experimentalistische Hedonisten, die am ehesten bestrebt sind, sich im Alltag umweltbewusst zu verhalten, führen an, dass sie ihren Prinzipien im Alltag nicht immer gerecht werden. Während beide Gruppen dies bedauern, zeigt sich bei den Materialistischen Hedonisten, dass Umweltschutz als soziale Norm nicht zweifelsfrei gesetzt ist. Im Gegenteil: Keinesfalls möchte man durch zuviel Umweltschutz auffallen und als "Ökotyp" gelten. Entsprechend entscheidet man hier nach dem Mehrheitsprinzip, d. h. wenn sich alle anderen auch an entsprechende Vorgaben halten, ist man grundsätzlich auch bereit, auf bestimmte "Bequemlichkeiten“ zu verzichten.

Beim Blick auf die Fortbewegungsmittel befinden sich die Jugendlichen aktuell qua Alter in der Situation, auf öffentliche Verkehrsmittel oder das Fahrrad zurückzugreifen bzw. zurückgreifen zu müssen. Für die Zukunft denken sie über Alternativen zum Auto durchaus nach, können sich aber 
nur bedingt vorstellen, zukünftig auf das Autofahren (völlig) zu verzichten. Das persönliche Mobilitätsverhalten künftig der Umwelt zuliebe zu verändern ist für die meisten Jugendlichen keine Option, insbesondere wenn man fürchtet, damit in eine Außenseiterrolle zu geraten.

( Also ich versuche auf jeden Fall nicht alles zu verdrecken. Ich probiere, wo ich kann mitzuhelfen. (weiblich, 16 Jahre, Sozialökologische)

So schwer ist Umweltschutz nicht. Die meisten Leute denken ja sofort, dass man sich Solarzellen anschaffen muss. Aber nein, das muss man gar nicht. Man braucht nur viel mit den Öffentlichen zu fahren oder Fahrgemeinschaften zu bilden. Da kann man auch wieder Sprit sparen. Oder man fährt mit dem Fahrrad. Ist das so schwer?! (weiblich, 16 Jahre, Experimentalistische Hedonisten)

( Wir könnten das jetzt so machen, wir gehen lieber duschen anstatt baden, solche kleinen Dinge. Zum Beispiel fahre ich jetzt auch Fahrrad und Bahn und nicht Auto. (männlich, 14 Jahre, Materialistische Hedonisten)

(7) Also ich bringe meinen Müll immer in meine Mülltonnen. Ich würde auch niemals ein Kaugummi einfach auf die Straßen werfen. Dafür gibt es Mülltonnen, und wenn gerade keine Mülltonne da ist, dann packe ich den in meine Tasche, bis ich an der nächsten Mülltonne bin. (weiblich, 17 Jahre, Materialistische Hedonisten)

( So Müll trennen, recyceln und so, das machen wir immer. Wenn ich draußen bin, schmeiße ich auch ungern Sachen auf den Boden. Ich suche immer erst mal einen Mülleimer. Ich mache es dann schon auch, das gebe ich zu, aber nur, wenn ich schon eine halbe Stunde mit dem Ding in der Hand herumlaufe und keinen Mülleimer finde. Duschen tu ich sowieso so kurz wie möglich. Und baden tut sowieso richtig viel Wasser verschwenden (männlich, 15 Jahre, Adaptiv-Pragmatische)

( Das finde ich schon auch wichtig. Also manchmal vergisst man es natürlich total und lässt dann das Licht an und dann denkt man, oh, okay, oder man ist zu bequem, um dann irgendwie doch zu laufen. (weiblich, 16 Jahre, Sozialökologische) 
(7) Ich werfe auch Müll auf die Straße, das macht eigentlich, glaube ich, jeder. Was mir schon Gedanken macht ist Auto fahren. Ich weiß nicht, wenn ich jetzt größer bin und aufs Autofahren verzichten will, ich glaube, das wird nicht so viel bringen. Ich bin einer von Milliarden, die das machen. Und nicht weil einer was macht, wird sich was ändern. Das denke ich halt immer. Oder weniger Müll produzieren. Natürlich hilft das ein bisschen, aber das ist so minimal eigentlich. Darum macht man sich jetzt nicht so viel Gedanken darüber. Außer man ist wirklich so ein Ökotyp oder eine Ökotante, dann schon. Wenn man so ganz normal ist, dann eigentlich nicht so. (männlich, 15 Jahre, Materialistische Hedonisten)

( Oh Gott. Also ich trenne weder meinen Müll, noch achte ich da irgendwie anders drauf. Und ich finde das ganz ganz furchtbar, dass ich so bin. Also ich würde es gerne. Ich würde echt gerne mehr auf meine Umwelt achten, aber ich bin zu faul. (weiblich, 17 Jahre, Experimentalistische Hedonisten)

\section{Jugendliche fragen sich, ob Umweltschutz wirklich „etwas bringt".}

Trotz generellen Commitments für den Umweltschutz im Alltag sind die Jugendlichen unsicher, ob ihre Aktivitäten tatsächlich „etwas bringen". Gerade diejenigen, denen Umweltschutz weniger wichtig ist, würden ihr Verhalten nur ändern, wenn sie wüssten, dass sie damit tatsächlich etwas Positives bewirken könnten oder das Gefühl hätten, Teil der Mehrheit zu sein: Wenn z. B. alle auf das Auto verzichten würden, wäre man gern dabei. Ansonsten möchte man hier und insbesondere beim Fleischkonsum nicht zurückstecken und sich schlimmstenfalls dadurch selbst ausgrenzen. Selbst die Sozialökologischen und Experimentalistischen Hedonisten, die eine besonders hohe Engagementbereitschaft zeigen, sind skeptisch, ob ihr eigenes Engagement wirklich etwas verändern kann, wenn sich alle anderen sperren. 
( Ich denke immer so, das bringt bestimmt nichts, wenn ich das jetzt mache, weil man kann es zwar weitergeben an Leute und so, aber das bringt dann ja nichts, dass ich dann da irgendwo da rumhocke und mir da Gedanken darüber mache, wenn die anderen es nicht umsetzen. (weiblich, 17 Jahre, Sozialökologische)

(1) Es ist so ein Ding, wenn die Mehrheit da mitmacht, dann könnte man selber da auch mit einsteigen. So als ein Einzelner kannst du jetzt nicht wirklich viel bewirken. Wenn da alle mitmachen würden, dann hat natürlich jeder auch die Motivation, weil das bringt wirklich was. Es ist für einen selber natürlich auch ein gutes Gefühl, wenn er weiß, $j a$, du hilfst dadurch der Umwelt. Aber wenn du natürlich im Hinterkopf behältst, dass sich trotzdem nicht viel ändern wird, weil der Rest so konsumstrebig lebt, dann ist es halt doch schon ein Knick für einen selbst. (männlich, 16 Jahre, Adaptiv-Pragmatische)

(7) Man kann natürlich sagen, ich fahre kein Auto. Dann bin ich aber einer der - was weiß ich wie vielen, die das machen. Das ist jetzt vielleicht blöd, das so zu sagen, aber es wird nichts ändern, wenn ich das jetzt mache. Weil halt jeder eigentlich ein Auto fährt. Und ich denke mal, das ist keine Sache, die sich über 100 Jahre direkt ändern könnte. Also 100 Jahre vielleicht schon, aber so 50 Jahre denke ich nicht, dass das reichen würde. (männlich, 17 Jahre, Adaptiv-Pragmatische)

Engagement für den Umweltschutz wird häufig mit Spenden assoziiert. Dabei legt man Wert darauf, dass entsprechende Anbieter "seriös" sind. Bei persönlichem Engagement in Organisationen legt man Wert darauf, "an den richtigen Stellen“ etwas bewegen zu können. Häufig werden diese Argumente auch herangezogen, um zu erläutern, warum man sich kein weiteres Engagement vorstellen kann - insbesondere bei Prekären und Materialistischen Hedonisten.

Spenden könnte man, aber das würde echt nichts helfen. Wenn ich jetzt zum Beispiel 100 Euro dahin spende, dann wären jetzt 100 Euro für mich weg, und da würde sich null dran ändern so. Man müsste da echt schon so mit Millionen oder so hin kommen. Aber wirklich dahin, aber 
alleine schon, wenn du dahin fährst, da geht schon die Hälfte von dem Geld weg. (männlich, 15 Jahre, Prekäre)

[int: Was meinst Du? KönNte es Vielleicht auch Spass machen, DA IRGENDWAS AKTIV ZU TUN?] Bestimmt. Auf jeden Fall. Ich finde nur, es ist schwer zu wissen, welche Organisation die richtige ist. Man weiß es nicht. Wie viele Organisationen gibt es, die sagen: Schickt uns Geld für die Kinder von Afrika? Und am Ende kommt es nicht mal an. Also ich weiß nicht. Wenn, dann will ich den Chef wirklich höchst persönlich sprechen. Und ich will wissen, dass es wirklich seriös ist. (weiblich, 16 Jahre, Materialistische Hedonisten)

\section{Fehlende Zeit wird als Hauptgrund genannt, sich nicht (noch) intensiver für Umweltschutz zu engagieren.}

Ein aktives, umfassenderes Engagement für den Umweltschutz, z. B. bei Organisationen wie Greenpeace oder im Rahmen eines Freiwilligen Ökologischen Jahres, wird nur selten angesprochen. Aktuell stehen - neben der fehlenden erlebten Handlungswirksamkeit - auch die mangelnden zeitlichen Ressourcen oder organisatorischen Rahmenbedingungen dem freiwilligen Engagement im Weg. Aber diejenigen, die dem Engagement für den Umweltschutz grundsätzlich offen gegenüber stehen, können sich auch gut vorstellen, dass dies Spaß machen würde und sich dabei das Gefühl einstellen könnte, etwas Sinnvolles zu tun.

Aber auch die Jugendlichen, die sich ein längerfristiges Engagement für den Umweltschutz vorstellen können, vertagen es auf später, wenn man erwachsen ist. Dann - so die mehrheitliche Vermutung - hätte man endlich mehr Zeit und vor allem die ökonomischen Ressourcen zur Verfügung, die die entsprechenden Verhaltensweisen und auch Freiheiten bei Konsumentscheidungen erlauben.

( Ich war mal bei Greenpeace. Aber da war ich noch etwas jünger. Jetzt habe ich nicht mehr genug Zeit. (weiblich, 16 Jahre, Adaptiv-Pragmatische) 
(1) Ich will später vielleicht mal nach China und dort ein paar Umweltsachen klären. Denn Deutschland ist auf Platz 1 der erneuerbaren Energien in der ganzen Welt. Und selbst ich habe jetzt so viel kritisiert, und dann schaut man nach China und die bomben die Luft voll mit Kohlendioxid und solchen Sachen. Und ich muss das einatmen. Das müssen die atmen. Die sind krank von dem ganzen Smog. Ich glaube, Peking wäre so eine schöne Stadt, wenn dieser Smog nicht wäre. Und da denke ich so, würden die mal einen richtigen Filter einbauen, wäre das auch mal nicht schlecht. (weiblich, 16 Jahre, Experimentalistische Hedonisten)

( Und ich finde das auch so schrecklich, wenn man den Regenwald abholzt. Ich würde das jetzt sofort stoppen, kann man halt diese Möbel nicht mehr kaufen, Pech gehabt, dann kauft man sich halt andere Möbel von anderen Bäumen, die man nachpflanzt. Ich glaube, ich werde mir auch als Erwachsener so ein Stückchen davon kaufen, das kann man ja. (männlich, 14 Jahre, Materialistische Hedonisten)

\subsection{Klimawandel}

\section{Klimawandel ist für Jugendliche etwas, das vor allem in entfernten Teilen der Welt stattfindet.}

Beim Stichwort "Klimawandel“ können die meisten Jugendlichen einige zentrale Aspekte beschreiben. Genannt werden beispielsweise die globale Erwärmung und deren Auswirkungen auf die Polkappen, das Ansteigen der Meeresspiegel, die Rodung der Regenwälder, das Verschwinden der Ozonschicht, extreme Wetterverhältnisse und Ernteausfälle. Vereinzelt werden auch soziale Auswirkungen auf verschiedene Regionen erwähnt, z. B. Klimaflüchtlinge.

Die einzelnen Puzzleteile zum Stichwort „Klimawandel“ fügen sich für die Jugendlichen aber nicht zu einem schlüssigen Ganzen zusammen. Für sie bleibt unklar, wie die verschiedenen Faktoren zusammenwirken und welche Entwicklungen welche Folgen haben könnten. Die Informationen 
aus Schule und Medien, die gleichzeitig die einzigen Bezugsquellen sind, erscheinen widersprüchlich und nicht klar nachvollziehbar.

Klimawandel ist zudem etwas, was aus Sicht der Jugendlichen - im Unterschied zum Umweltschutz - nicht vor der eigenen Haustür stattfindet, sondern eher auf anderen Kontinenten bzw. an der Peripherie der Weltkarte (Antarktis, Sibirien, Mikronesien) - und dies auch "erst" in einigen Jahrzehnten.

Typische Zitate zur Illustration

[INT: UND WAS DENKST DU, WENN DU KLIMAWANDEL HÖRST?] An den Treibhauseffekt und so. Und auch an die Eisbären und die Eisschollen, die schmelzen, an so was alles. (weiblich, 15 Jahre, Adaptiv-Pragmatische)

( Der Klimawandel ist natürlich auch ein großer Konflikt. Wenn der Meeresspiegel, ansteigt, und die ganzen Flüchtlinge aus den Ländern kommen, die überflutet werden. Wir reden in der Schule auch viel über den Klimawandel, auch in anderen Fächern wie Englisch. Ich denke, das wird ein großer Konflikt werden, vor allem wegen der Flüchtlinge. (weiblich, 17 Jahre, Adaptiv-Pragmatische)

( Ja, es wird sich bestimmt etwas verändern, auch vom Klimawandel, glaube ich. Zum Beispiel die Ozonschicht geht jetzt langsam auch immer mehr kaputt. Es wird dann halt auch immer gefährlicher in der Sonne. Das ist auch für mich ein Problem, was werden könnte, also schlimmer noch. Bisher ist es schon relativ schlimm in manchen Regionen und Gebieten. (männlich, 15 Jahre, Materialistische Hedonisten)

(7INT: UND WENN DU KLIMAWANDEL HÖRST?] / ch glaube nicht, dass sich das noch ändern wird so großartig. Es sei denn, wir stehen wirklich kurz vor irgendeiner schlimmen Katastrophe. Ich habe mal gehört, ich weiß absolut nicht, ob es stimmt oder nicht, dass uns eine Eiszeit bevorsteht wegen dem Klimawandel. Was ja eigentlich komisch ist, weil es wird ja immer wärmer. (weiblich, 16 Jahre, Sozialökologische)

8 Dass man den Regenwald abholzt und dass es nicht mehr lange dauert, bis der komplette Regenwald abgeholzt ist und dass der Klimawandel dann rasant eintritt. Ich weiß jetzt gar nicht, ob die Temperaturen 
steigen oder sinken. Dass dann vielleicht wieder eine Eiszeit ist oder es sehr heiß ist und dann die Wirtschaft auch nicht mehr gut funktionieren wird. Man kann im Winter zum Beispiel Nahrungsmittel nicht anbauen und herstellen, im Sommer kann man andere Sachen nicht mehr machen. Das würde verheerende Folgen haben. (männlich, 15 Jahre, Materialistische Hedonisten)

(7) Man hat ja geredet, dass 2050 wieder eine Eiszeit auf uns zukommen wird. Am Anfang dachte ich: ,Oh scheiße, Eiszeit, was machen wir?' Weil 2050 werde ich, glaube ich, noch erleben. Ich glaube aber, dass durch unsere Technik und alles, das nicht so extrem wird. Es kann natürlich sein, dass irgendwelche Wetterbedingungen jetzt so keine Ahnung, ein Komet die ganze Zeit über uns schwebt, dass die Wetterbedingungen auf der einen Seite, dass das halt richtig krass wird, müssen wir uns halt anpassen. Ich glaube, das Wetter wird noch die größte Hürde, die jetzt auf uns zukommt. (männlich, 14 Jahre, Materialistische Hedonisten)

\section{Wer wenig informiert ist, zeigt sich gegenüber dem Klimawandel eher gleichgültig.}

Vor allem bildungsferne Jugendliche, die wenig über den Klimawandel wissen oder einzelne Aspekte nicht in einen Wirkungszusammenhang bringen können, begegnen dem Thema emotionslos und indifferent. Sie denken kaum über die Folgen des Klimawandels für sich und kommende Generationen nach.

Vereinzelt werden von dieser Gruppe eindrückliche Naturphänomene, die man im Internet gesehen hat, als Folge des Klimawandels erwähnt. Diese werden aber eher als interessante News berichtet und nicht weiter als Problem beschrieben.

(7INT: WAS GEHT DIR DURCH DEN Kopf, WENn DU DAS WORT "KLIMAWANDEL" HÖRST?] Boah, die ganzen Autos glaube ich, die das Ganze verursachen. Die Heizungen, die im Sommer laufen. Ja, das eigentlich. (weiblich, 16 Jahre, Konservativ-Bürgerliche) 
(In der Sommerzeit kann man jetzt viel mehr machen. Schwimmen gehen, wenn das Wetter gut ist, kann man Fußball spielen gehen und Shisha rauchen im Park, solche Sachen. (männlich, 17 Jahre, Materialistische Hedonisten)

(2) [INT: Fällt Dir WAS EIN, WENn Du „KLIMAWANDEL“ HÖRSt?] Klimawandel? Habe ich noch nie gehört. (weiblich, 15 Jahre, Materialistische Hedonisten)

(7) [INT: WAS GEHT DIR DURCH DEN Kopf, WenN DU KLIMAWANdel HÖRST?] Wenn ich das Wetter heutzutage sehe, dann war gestern Sonne, heute dann mal wieder Regen. [INT: NocH IRGENDWAS?] Eigentlich nicht. (männlich, 14 Jahre, Prekäre)

[INT: UND WENN DU AN KLIMAWANDEL DENKST, IST DAS EIN THEMA FÜR DICH?] Nee, ich kenne mich eigentlich nicht so gut damit aus. (männlich, 16 Jahre, Materialistische Hedonisten)

(1) [INT: WAS DENKST DU ÜBER DEN KLIMAWANDEL?] Das ist mir eigentlich egal. (weiblich, 17 Jahre, Materialistische Hedonisten)

( Hier in Deutschland hat sich das Klima in letzter Zeit ganz schön verändert. Das habe ich gelesen. Es gab auch einen Tornado, das war eines der meistgesehenen Videos in YouTube. Ich weiß nicht, welches Dorf das war. (männlich, 15 Jahre, Adaptiv-Pragmatische)

(Ja so ans Globale denke ich. Also dass jetzt der Meeresspiegel steigt, es heißer wird. Das zum Beispiel. In Russland ist jetzt so wie in Deutschland, die Kälte. Weil früher war der Schnee einfach höher als wenn du stehst und deine Hände hoch hebst, so hoch war der Schnee. Und das ging bis zum vierten Stock damals, so krass war das. (männlich, 15 Jahre, Prekäre)

\section{Einige bezweifeln, dass der Klimawandel wirklich stattfindet.}

Nicht wenige Jugendliche, auch die formal höher Gebildeten, zeigen sich verunsichert, ob der Klimawandel wirklich genau vorausgesagt werden kann, ob die Zusammenhänge alle richtig beschrieben und die 
drohenden Veränderungen nicht übertrieben dargestellt werden. Diese Einschätzung vertreten teilweise auch Jugendliche, die sich nach eigenem Dafürhalten intensiv mit dem Thema beschäftigt haben.

Jugendliche äußern hier (wie auch in anderen Zusammenhängen, z. B. Finanzkrise) Zweifel, ob aktuelle und bevorstehende Ereignisse von Medien und Schule nicht dramatisiert und stark übertrieben werden.

(1) Es gibt Klimaerwärmung und die Gletscher schmelzen auch. Dadurch wird der Wasserspiegel weiter steigen und so weiter, dadurch gehen die Inseln unter, dann geht wieder mehr Land verschwunden. Und dafür gibt es dann auch wieder mehr Erdbeben, dadurch, dass die Plattenverschiebung anders wird. Das hängt auch zusammen und klar ist es auch wichtig und wir müssen garantiert etwas für den Klimawandel tun, aber es ist halt auch die Frage, inwiefern man auch den Klimawandel voraussagen kann. (weiblich, 17 Jahre, Adaptiv-Pragmatische)

(8) Es gibt so ein Buch, das heißt "Cool it!", glaube ich. Das habe ich begonnen zu lesen. Ich glaube, das ist von einem Statistiker. Der sagt, dass das jetzige Ansteigen der Temperatur ein natürlicher Rhythmus ist. Er behauptet auch, dass vieles falsch dargestellt wird. Das fand ich ganz interessant. (weiblich, 15 Jahre, Expeditive)

(8) Der Klimawandel ist ja nicht wirklich bewiesen. Ich glaube zwar auch dran, aber es ist halt nicht wirklich richtig bewiesen. Man weiß nicht, was für Zyklen jetzt sind, ob das vielleicht 100 Jahre sind, dann haben wir noch nicht mal lange genug gemessen, um diesen Zyklus herauszufinden. Aber ich vermute schon, dass es so etwas gibt. [INT: MACHT DIR DAS SORGE, SO ETWAS WIE DER KLIMAWANDEl?] Jein. Es macht mir Sorge, aber für uns ist es eigentlich noch uninteressant. (männlich, 17 Jahre, Konservativ-Bürgerliche)

(7) Erderwärmung kann ich jetzt nicht so beurteilen. Ich denke schon, dass ein bisschen was dran ist, aber es wird bestimmt so ein bisschen übertrieben immer. (männlich, 14 Jahre, Adaptiv-Pragmatische) 


\section{Die Alltagswahrnehmung von Wetter und Klima stimmt für Jugendliche nicht mit den benannten Folgen des Klimawandels überein.}

Die Jugendlichen vergleichen die Meldungen zum Klimawandel und zu steigenden Temperaturen mit ihren alltäglichen Erfahrungen. Ein kalter Sommer oder unterschiedliche Temperaturen in verschiedenen Regionen stimmen für sie dabei nicht mit den allgemeinen Aussagen zum Klimawandel überein. Ein Temperaturanstieg von zwei Grad Celsius ist weder im Alltag wahrnehmbar, noch kann man nachvollziehen, warum das so schlimm sein soll. Entsprechend sehen manche Jugendliche - vor allem Adaptiv-pragmatische Jugendliche und Materialistische Hedonisten den Klimawandel nicht als großes Problem. Die meisten Jugendlichen können sich daher kaum vorstellen, dass der Klimawandel für sie konkrete negative Folgen haben könnte.

Typische

Zitate zur

Illustration

Und mit dem Klimawandel ist auch so eine Sache. Man sagt zwar, es ist so warm, allerdings ist der Sommer ja nicht so warm. Es war jetzt das heißeste Wochenende seit soundsoviel Jahren, aber das war ein Wochenende, und der Rest des Sommers ist dann kalt. (weiblich, 17 Jahre, Adaptiv-Pragmatische)

( So was haben wir auch in der Schule, aber Klimawandel ist ja auch so was wie die Temperaturen hier. Ich finde es manchmal voll komisch, weil wir sind jetzt schon im Juli und es ist immer noch so kalt. Im Gegensatz dazu, in der Türkei sind es schon 45 Grad. Und wir haben hier gerade noch 18 oder 19 Grad. Sollte wärmer werden. (weiblich, 14 Jahre, Materialistische Hedonisten)

Wirklich realisieren kann ich das nicht, dass die Erde wärmer wird, wenn der Winter immer so kalt ist. Aber ich mach mir da schon Sorgen drum, wenn das dann ehrlich so passiert und diese Polarkappen da schmelzen und so. Ich meine, irgendwie sind wir dann ja anscheinend dran Schuld, und dass wir da irgendwie versuchen, was zu ändern. (weiblich, 15 Jahre, Sozialökologische) 
(1) Wenn die so sagen, dass es zwei Grad wärmer werden soll, denkt man immer: Das ist ja nicht wirklich so bedeutend. Aber was da so dahinter steckt, gerade in so Gletschergebieten, dass es sein kann, dass es schmilzt, in der Schweiz oder so ist das, wo sich der Gletscher zurückbildet, keine Ahnung. Und dass es halt dann wärmer ist. Aber so richtig einen Unterschied, gerade hier in Deutschland, macht das ja jetzt auch nicht. Es ist halt im Winter, dass es dann mal nicht schneien kann, weil es zwei Grad zu warm ist." (männlich, 14 Jahre, Adaptiv-Pragmatische)

\section{Jugendliche setzen auf technologische Entwicklungen bei der Bewältigung des Klimawandels.}

Für die Jugendlichen ist Klimawandel noch mehr als Umweltschutz ein Thema, dessen Bewältigung sie gerne "delegieren“ würden - „an Leute, die sich damit auskennen". Sie hoffen u.a. auf technologische Entwicklungen (Windkraft, Solarenergie, Elektroautos) im Rahmen einer konsequenten Energiewende, um den Klimawandel abzuwenden oder ihm zu entgehen. Ähnlich wie beim Umweltschutz sind sie sich aber nicht sicher, ob sich eine Katastrophe in Zukunft wirklich noch verhindern lässt. Bei den meisten Jugendlichen resultiert dies in einem abgeklärten Fatalismus: Klimawandel lässt sich offenbar nicht aufhalten, aber vermutlich muss man die Konsequenzen persönlich nicht mehr erleben. Manche Jugendliche äußern aber auch eine diffuse Angst, die sich vor allem auf die Folgen für die eigenen Nachkommen konzentriert.

( Darüber weiß ich, glaube ich, echt zu wenig. Also ich habe nur ganz furchtbar Angst davor, dass meine Enkelkinder da später ein Problem mit bekommen. Das war's (weiblich, 17 Jahre, Experimentalistische

Typische Zitate zur Illustration Hedonisten)

Klimaerwärmung, das ist ja auch gerade ein ganz großes Thema überall. Und dass sich jeder irgendwie mehr beteiligen sollte, weil das auch eine ernste Sache ist, die man auf keinen Fall ignorieren sollte. (weiblich, 16 Jahre, Experimentalistische Hedonisten) 
( Das Ding ist ja, wir haben nur diesen einen Planeten. Wir haben nur eine Chance. Und die werden wir garantiert verhauen. Und wenn man sich jetzt mal die Welt sich anguckt, ist es einfach so. (männlich, 17 Jahre, Adaptiv-Pragmatische)

(>) [INT: WAS FÄlLT DIR EIN, WENN DU DEN BEgRIFF KLIMAWANDEL HÖRST?] Eigentlich nur so Solar- und Windtechnik. Dass man dadurch halt neuen Strom gewinnt und halt nicht. Also eigentlich fällt mir nur ein, dass es so jetzt andere Möglichkeiten gibt, zum Beispiel Strom zu gewinnen. Das finde ich gut. (weiblich, 15 Jahre, Experimentalistische Hedonisten)

Ich würde es cool finde, wenn wir das, wenn wir wissen, okay, es wird bald passieren, dass die Erde untergeht, dass wir dann so weit fortgeschritten sind, dass wir mit Raumschiffen wegfliegen können. Also ich will nicht, dass die Menschheit ausstirbt, auch wenn ich dann nicht mehr leben werde. (männlich, 14 Jahre, Materialistische Hedonisten)

\section{Für eigene Aktivitäten gegen den Klimawandel fehlen den Jugendlichen oftmals das notwendige Wissen und konkrete Ideen.}

Engagement für den Klimaschutz kann man sich weniger gut konkret vorstellen als für den Umweltschutz. Das Problem bleibt trotz kontinuierlicher Präsenz in der Schule und den Medien abstrakt und die Konsequenzen nicht einzuordnen. Daher betonen die meisten Jugendlichen deutlich, dass sie nicht aktiv werden wollen. Es fehlt ihnen sowohl an Wissen als auch an Antrieb oder die Ideen dafür.

Vereinzelt richten Jugendliche ihr Alltagshandeln aber klimafreundlich aus oder engagieren sich gar aktiv gegen den Klimawandel. Sie versuchen beispielsweise weniger Strom zu verbrauchen, die Heizung herunterzudrehen oder Fahrrad statt Auto zu fahren. Einige Jugendliche möchten in Zukunft mit Elektroautos fahren, Solarenergie nutzen oder sogar beruflich oder ehrenamtlich im Bereich Klimaschutz aktiv werden. Aber genau wie beim Umweltschutz befürchten die Jugendlichen auch hier, 
dass das Engagement Einzelner solange wirkungslos bleibt, bis sich nicht eine "kritische Masse" entschließt mitzumachen.

( Was den Klimawandel angeht, hab ich eigentlich gar keine Ideen. Aufhalten kann man es sowieso nicht mehr. Natürlich gibt es immer so die Sachen, dass man weniger fliegen sollte und alles, aber ich glaube, wenn man selber das entscheidet und nur man selber das macht - das ist natürlich auch gut, dann hat man auch kein schlechtes Gewissen mehr, aber solange es nicht ein paar mehr Menschen machen, bringt es sowieso nichts" (weiblich, 16 Jahre, Expeditive)

( Ja, ich denke da schon ab und zu drüber nach. Und also ich benutze kaum Licht hier im Zimmer, auch wenn ich auf das Klo gehe nachts. Fernseher aus. Also eigentlich immer alles aus machen, was gerade nicht gebraucht wird. (weiblich, 17 Jahre, Konservativ-Bürgerliche)

( Man könnte sich auf jeden Fall engagieren, dann könnte man sicherlich auch selber mit dem Elektroauto fahren. Also hier geht es jetzt nicht, aber wenn man ein Haus hätte, eine Solaranlage auf dem Dach haben und sowas. Und größtenteils die Energie eben selber produzieren. Das geht auf jeden Fall. Und wenn das jeder machen würde, dann würde es auf jeden Fall besser werden. [INT: KANNST DU DIR AUCH VORSTELLEN, DICH IN DER ART UND WEISE IRGENDWIE ZU ENGAGIEREN UND DA MEHR ZU MACHEN?] Ja, kann ich mir auf jeden Fall vorstellen, wenn ich zum Beispiel irgendwann ein Haus haben sollte, dann denke ich, werde ich auf jeden Fall auch eine Solaranlage haben und sowas. Und halt unnötige Sachen zu vermeiden, wie zum Beispiel den Wasserhahn laufen zu lassen unnötig und sowas. (männlich, 16 Jahre, Materialistische Hedonisten)

(7) [INT: ABER INWIEWEIT IST DAS DENN WICHTIG FÜR DICH, ALSO WÜRDEST DU SAGEN, BIST DU DA AUCH SELBER AKTIV, DA WAS DAGEGEN ZU MACHEN?] Wenn ich erwachsen bin, dann. Ich habe auch schon überlegt, ob ich so einen Job mache, also ich will, wenn ich erwachsen bin, dann auch beitragen. [INT: WAS WÜRDE DIR DENN DA SPASS MACHEN, AN WAS DENKST DU DENN ODER AN WAS HAST DU SO GEDACHT?] Ich glaube, so eine Mitgliedschaft einzutreten wäre cool und da auch 
immer was zu spenden, ja, würde ich glaube ich auch mal machen. Kommt natürlich auch drauf an, wegen Geld. (männlich, 14 Jahre, Materialistische Hedonisten)

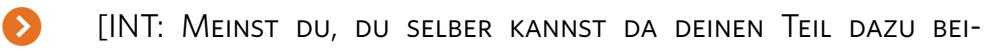
TRAGEN, DASS ES BESSER WIRD?] Ich könnte so eine Organisation gründen, aber da gibt es schon genug von. Ich würde es jetzt nicht machen, ich wäre einfach auch zu faul dafür. (männlich, 15 Jahre, Adaptiv-Pragmatische)

[INT: KANNST DU DIR VORSTELLEN, SELBER DA AKTIV ZU WERDEN?] Nein! Das kann ich nicht. [INT: WARUM WÜRDEST DU DAS NICHT MACHEN WOLLEN?] Ich kann partout nicht einschätzen, was richtig ist und was falsch ist. (weiblich, 14 Jahre, Experimentalistische Hedonisten)

\subsection{Kritischer Konsum}

\section{Der Begriff „kritischer Konsum" ist Jugendlichen weniger bekannt als die Begriffe "Umweltschutz" oder "Klimawandel“.}

Nur wenige Jugendliche verbinden mit dem Begriff "kritischer Konsum" eine konkrete Bedeutung oder zugehörige Handlungsweisen. Teilweise gehen ihre Assoziationen in Richtung gesunde Ernährung, Medienkonsum, Umweltschutz oder Sparsamkeit und treffen damit lediglich spezifische Aspekte des Themas. Am ehesten noch haben sich schon Sozialökologische und Expeditive Jugendliche mit Fragen des kritischen Konsums auseinandergesetzt. Jugendliche dieser beiden Lebenswelten können beschreiben, dass es grundsätzlich darum geht, wie konsumiert wird und in welchem Ausmaß.

Typische

Zitate zur Illustration
[INT: SAGt diR DER BegrifF "KRITISCher Konsum" etwas?] Konsum ist ja so etwas, was man einnimmt, oder nicht? (weiblich, 16 Jahre, Materialistische Hedonisten) 
(1) Ja man muss halt darauf achten, wie zum Beispiel der Regenwald abgeholzt wird, wie die Plantagen so. Wir sind halt eine sehr konsumgierige Gesellschaft, also denken nur ans Konsumieren, Konsumieren, Konsumieren, aber achten halt auch nicht auf die Umstände, wenn man halt Fakten liest, dass teils über tausend Schweine am Tag sterben, nur damit wir unseren Fleischhunger stillen. Das sind halt ungeheure Kosten. Und teils auch viel Natur, man fragt sich halt, wie lange das jetzt noch gut gehen soll. Wenn die Schweine dann aussterben oder die Kühe, dann ist das natürlich auch ein Problem. (männlich, 16 Jahre, Adaptiv-Pragmatische)

(7INT: WAS GEHT DIR DURCH DEN KOPF, WENN DU "KRITISCHER KONSUM" HÖRST? HAST DU EINE IDEE, WAS DAS SEIN KÖNNTE?] Das ist bestimmt so was mit Lebensmitteln oder so was. Kritischer Konsum? Haben wir so was in Deutschland? Ich glaube nicht, ne? (männlich, 15 Jahre, Prekäre)

(7) [INT: WenN ICH "KRITISCHER KONSUM" SAGE, WAS FÄlLt DIR DAZU EIN?] Konsum in der Form, dass man vielleicht, wenn man ein Smartphone benutzt, das ein bisschen kritisch hinterfragt, wie viel man es benutzt und wie man es benutzt. (weiblich, 17 Jahre, Expeditive)

( Kritischer Konsum? Da geht mir durch den Kopf, dass... Eigentlich fällt mir da nichts ein. (weiblich, 17 Jahre, Materialistische Hedonisten)

Jenseits der Begrifflichkeit sind Jugendlichen jedoch die Debatten um billige Produkte und menschenunwürdige Produktionsbedingungen durchaus bekannt. Vor allem Befragte aus Lebenswelten, in denen der regelmäBige Kauf aktueller Mode sehr wichtig ist (inbesondere Materialistische Hedonisten) benennen aktiv die Herstellungs- und Vertriebsproblematik billiger Modemarken wie Primark oder Kik.

Somit ist die Präsenz dieses Themas nicht unbedingt abhängig vom grundsätzlichen Interesse an Fragen zu sozialer und ökologischer Gerechtigkeit, sondern hängt eng damit zusammen, wie wichtig ein bestimmter Konsumbereich generell ist: Gerade die Jugendlichen, die viel und häufig bei sog. „Billigmarken“ einkaufen, sahen sich bereits mit entsprechender Berichterstattung oder Protestaktionen (z. B. bei einer Filialeröffnung) über Missstände bei der Produktion konfrontiert. Auch 
wenn sie ihr aktuelles Kaufverhalten nicht ändern können oder wollen, beschäftigt sie es, dass in „ihrem" Laden Menschen unter sehr schlechten Bedingungen arbeiten müssen.

( Ja, manchmal schon. Weil zum Beispiel Primark, das ist ja eigentlich nur Kinderarbeit. Und voll viele gehen ja da shoppen, weil es so billig ist. Aber manchmal denke ich auch daran und denke, Mädel, du könntest auch eines von den Kindern sein. Weil die Kinder bekommen ja nicht so viel Geld von da, das verdiente Geld von Primark. Voll wenig bekommen die Kinder dort. (weiblich, 14 Jahre, Materialistische Hedonisten)

Adaptiv-Pragmatische und Experimentalistische Hedonisten nennen bei der Problematik rund um billigen und global gesehen schädlichen Konsum häufiger Themen aus dem Bereich Ernährung, z. B. Abholzung des Regenwaldes für Lebensmittel oder Massentierhaltung. Primär geht es ihnen dabei aber um die eigene Gesundheit und nicht unbedingt um die langfristigen Folgen für die Umwelt.

(7INT: UND WAS DENKST DU, WENN DU KRITISCHER KONSUM HÖRST?] SO an Hamburger und so, ich weiß nicht. Wenn man da zu viel isst, ich weiß nicht, Zucker, ich weiß nicht. Ich denke da irgendwie an Ernährung. (weiblich, 15 Jahre, Adaptiv-Pragmatische)

[INT: Jetzt haben Wir Noch den Begriff Kritischer Konsum] Oh. [INT: WORAN DENKST DU DA SO?] Da denke ich an Ernährung in erster Hinsicht und dann eben diese ganze technische Entwicklung, Medienkonsum und so. (weiblich, 17 Jahre, Experimentalistische Hedonisten)

\section{Wenn Jugendlichen der Begriff "kritischer Konsum" erläutert wird, bewerten sie diesen überwiegend positiv.}

Wenn die Jugendlichen die Idee des kritischen Konsums erläutert bekommen, fallen ihnen zahlreiche Beispiele ein, wie sie diesen in ihrem Alltag realisieren könnten, z.B. durch den Kauf biologischer, regionaler oder fair-gehandelter Produkte. 
Als Motivation für kritischen Konsum wird von den Jugendlichen in diesem Zusammenhang vor allem Kinderarbeit angeführt. Andere ethische und arbeitsrechtliche Aspekte, wie z. B. geringe Löhne für erwachsene Arbeiterinnen und Arbeiter oder ungenügende Sicherheitsmaßnahmen am Arbeitsplatz werden dagegen eher selten genannt. Trotzdem wünscht man sich ganz allgemein faire Arbeitsbedingungen für die Menschen, die die Kleidung herstellen.

Ach so. Das ist schon ziemlich hart, wenn man das so hört, wenn bei Primark Klamotten aus Kinderhänden stammen. Und dass das so eigentlich mit der Welt gar nicht laufen sollte, weil die leiden müssen, damit wir schön sind. Das finde ich nicht gut. (weiblich, 15 Jahre, Sozialökologische)

[INT: HAST DU SCHON MAL ETWAS ÜBER DIE BEDINGUNGEN GEHÖRT, WIE KLAMOTTEN HERGESTELLT WERDEN?] Ich habe mal gehört, dass in China die Kinder dafür arbeiten müssen. Das finde ich nicht so gut. (weiblich, 15 Jahre, Materialistische Hedonisten)

\section{Viele Jugendliche sehen ihre begrenzten finanziellen Mittel als wesentlichen Hinderungsgrund für kritischen Konsum.}

Kritischer Konsum ist aus Sicht der Jugendlichen vor allem eine Frage der finanziellen Möglichkeiten. Sie wissen, dass faire Produktionsbedingungen teurer sind. Konsumverzicht wird von den Jugendlichen aber kaum thematisiert. Nur vereinzelt erwähnen sie, dass kritisch zu konsumieren auch bedeuten kann, das bisherige Konsumverhalten zu hinterfragen, Maßlosigkeit zu vermeiden und sich in Sparsamkeit zu üben. Das betrifft dann nicht nur Lebensmittel, Kleidung, sondern auch den Stromverbrauch und Medienkonsum. Vor allem Materialistische Hedonisten möchten sich hier nicht einschränken.

(7) [INT: WeNN DU ZUM BEISPIEL KLEIDUNG TRÄGST, IST ES WICHTIG FÜR DICH, WO DIE PRODUZIERT WURDE?] Ja, also klar, es ist natürlich wichtig dass es den Arbeitern gut geht. Aber was will man dagegen machen. 
Wenn es billig sein soll, dann muss es halt so sein. Wenn man viel Geld ausgibt, dann wird es bestimmt irgendwo besser produziert. Aber wenn man jetzt nicht so viel Geld zur Verfügung hat, dann kann man eigentlich nichts anderes machen. (männlich, 16 Jahre, Materialistische Hedonisten)

( Ich finde es schon wichtig, auf so etwas zu achten, aber man will ja die geringen Preise bezahlen. Man will ja nicht unbedingt viel bezahlen und das ist einfach so ziemlich der einzige Weg, es so billig zu machen. Und ich finde, solange man nicht bereit ist, mehr zu bezahlen, wird es auch diesen kritischen Konsum nicht geben. Die so etwas machen, sind welche die viel Geld haben oder zu viel Geld, dass sie es sich leisten können. (männlich, 17 Jahre, Konservativ-Bürgerliche)

\section{Vereinzelt haben Jugendlichen bereits Aspekte des kritischen Konsums in ihren Alltag integriert - sowohl aus ex- wie intrinsischer Motivation.}

Teilweise haben sich die Jugendlichen für einen vermehrten Konsum fair gehandelter Kleidung und biologischer Lebensmittel entschieden. Sie wollen damit ein Vorbild für andere Jugendliche sein und meinen, dass irgend jemand damit anfangen müsse. Dies trifft insbesondere auf Sozialökologische Jugendliche zu.

Andere Jugendliche beschreiben, dass sie sich mit dieser Art des Konsums "einfach besser fühlen". Gerade Adaptiv-Pragmatische meinen zudem, dass eine vegetarische oder vegane Lebensweise auch aktuell eine Art Life-Style-Trend ist, bei dem sie dabei sein möchten.

Typische

Zitate zur Illustration
Man kann halt einfach aufhören, die Klamotten zu kaufen. Man sagt ja immer, nur einer, das macht keinen Unterschied, aber es macht irgendwie doch einen Unterschied, weil wenn du es nicht machst, dann machen es vielleicht deine Freunde auch nicht. Ich denke schon, dass man als Einzelperson etwas machen kann. Vielleicht nicht den Riesenunterschied, aber vielleicht einen Anfang. (weiblich, 17 Jahre, Sozialökologische) 
(>INT: IST ES DIR ZUM BEISPIEL WICHTIG, WO DIE KLEIDUNG, DIE DU TRÄGST, PRODUZIERT WIRD?] Eigentlich schon, weil man ja viel hört, mit dieser Kinderarbeit. Und das ist ja nicht so gut. Oder ich versuche halt schon, mich ein bisschen darüber zu informieren, dass es halt nicht gerade so billig ist, sage ich mal. Dass das quasi nicht gut hergestellt werden konnte. (weiblich, 15 Jahre, Sozialökologische)

I Ich probiere halt, nicht so viel Fleisch zu essen. Wir haben auch so eine vegetarische Wurst. Da gibt es ja jetzt zurzeit richtig viel Auswahl und es ist auch ziemlich lecker. Also ich probiere da schon, drauf zu achten. Und bei Obst drauf zu achten, dass es aus Deutschland kommt. Wenn es jetzt nichts gibt, das aus Deutschland kommt, dann kaufe ich es trotzdem, aber wenn es halt eine Variante aus Deutschland zu kaufen gibt, dann Deutschland. (weiblich, 15 Jahre, Adaptiv-Pragmatische)

(7) Fleischkonsum ist bei uns zumindest sehr wenig. Meine Mutter ist auch so ein bisschen auf diesem - ich will nicht sagen Hippie-Trip, aber sie guckt halt immer, dass es Fleisch aus dem Bioladen ist. Es ist halt sehr teuer, aber dafür gibt es auch nur ein-oder zweimal in der Woche Fleisch. Ich bin auf jeden Fall gegen diese Massentierhaltung, weil man braucht nicht so oft Fleisch in der Woche. Mir reicht das vollkommen ein oder zwei Mal. Und es schmeckt auch viel besser, wenn man so ein frisches Stück Fleisch hat als so ein abgepacktes aus der Tiefkühle. Ich denke, da kann man ein bisschen mehr nachdenken, was man isst, und vor allem wie viel. (männlich, 14 Jahre, Adaptiv-Pragmatische)

\section{Die Bereitschaft zu kritischem Konsum hängt von der Produktkategorie ab: Für verantwortlich hergestellte Lebensmittel würden Jugendliche eher einen höheren Preis bezahlen als für Kleidung.}

Lebensmittel für den heimischen Haushalt werden in der Regel noch von den Eltern gekauft. Wenn diese ökologische und fair gehandelte Produkte kaufen (z.B. Produkte aus dem Bioladen oder regional erzeugtes Fleisch), finden die Jugendlichen das gut. Und da Lebensmittel in der 
Regel günstiger sind als Kleidung, können sich die Jugendlichen auch vorstellen, zumindest hin und wieder selbst Bio-Produkte zu kaufen.

Zudem haben die meisten Jugendlichen mehr Vertrauen in die ökologische und regionale Herkunft von Lebensmitteln als in den Nachweis fairer Produktionsbedingungen von Kleidung. Hinzu kommt, dass aus ihrer Sicht auch gesundheitliche Aspekte für den Kauf von Bio-Produkten sprechen.

Typische Zitate zur |llustration

Also ich würde eher sagen, es ist mir eher wichtig, was für Lebensmittel ich kaufe, als die Tatsache, wo ich meine Kleidung kaufe, also es gibt diesen Fair Trade auf jeden Fall, aber bei dem Fair Trade steht auch nicht alles drauf. Das ist das Problem. Das habe ich auch mit meinem Bruder diskutiert, dass es nicht unbedingt so sein muss wie es halt draufsteht. Es steht nie irgendwo alles drauf. Und ja, ich bin auch bereit, ein bisschen mehr dafür zu bezahlen und ich bin auch in den Biomarkt, da bin ich auch bereit, ein bisschen mehr zu zahlen, dafür dass es dann Bio ist oder halt auch aus Deutschland kommt. Aber ich sage halt, bei Kleidung, ob ich irgendwo es bei Hollister kaufe, wo es teurer ist und wo es halt besser produziert sein muss, aber es ist trotzdem gleich produziert ist im Endeffekt, oder ob ich jetzt halt bei einer Billigmarke einkaufe, ist halt nicht so der große Unterschied. (weiblich, 17 Jahre, Adaptiv-Pragmatische)

$>$

[INT: UND AUCH WENN DU SO AN ESSEN DENKST, ACHTEST DU ZUM BEISPIEL DARAUF, WAS DU FÜR FLEISCH ODER SO KAUFST ODER OB DAS BIO-PRODUKTE SIND?] Neuerdings schon, aber auch erst so seit einem Jahr. Und ich bin jetzt auch keine Veganerin oder so, aber da achte ich schon sehr drauf, allgemein bei meiner Ernährung, hat aber auch was einfach mit meiner Gesundheit zu tun und gar nicht mal die Umstände. (weiblich, 17 Jahre, Experimentalistische Hedonisten)

(8) Obwohl mein Opa gesagt hat, dass man nicht immer glauben kann, wenn da "Bio" draufsteht, dass da $100 \%$ Bio drin ist oder dass die Kühe nur Gras essen auf der Weide und nicht Heu in der Scheune. (männlich, 15 Jahre, Materialistische Hedonisten) 
( Ich würde mich eigentlich gern nur von Bio-Sachen ernähren. Das würde ich gern, aber es ist halt dementsprechend teurer. Ich glaube, wir kaufen schon ein bisschen Bio, aber nicht alles. [INT: WEIL ES GESUND FÜR EUCH IST ODER WEIL DU DIR AUCH GEDANKEN MACHST, WIE ES HERGESTELLT WIRD? ODER WENN DU DIR KLAMOTTEN KAUFST, MACHST DU DIR DA GEDANKEn?] Bei Klamotten eigentlich nicht. Wenn ich etwas kaufe, was mir wirklich gefällt, dann kaufe ich das halt. Und was ich halt brauche. Ich kaufe ja nicht irgendwas. Aber eigentlich mache ich mir nicht so viele Gedanken darüber. (männlich, 15 Jahre, Materialistische Hedonisten)

Nur vereinzelt wird bei Bio-Lebensmitteln ein "unvertrauter" Geschmack als Hinderungsgrund erwähnt.

Ich mag keine Bio-Sachen, überhaupt nicht. [INT: WAS SCHMECKT DIR DARAN NICHT?] Also ich finde es schmeckt komisch. Ich mag das nicht. Also ich habe mal eine Bio-Melone gegessen, glaube ich war das, das konnte ich nicht Das war so ekelig, das schmeckt ganz anders, das mag ich nicht. Nee, das mag ich nicht. (weiblich, 17 Jahre, Materialistische Hedonisten)

Der Kauf fair hergestellter Kleidung scheitert vor allem an den Präferenzen der Jugendlichen für bestimmte Stile und Marken sowie an ihren begrenzten finanziellen Mitteln. Aber auch der Aufwand für die Informationsbeschaffung, ob und welche Kleidungsstücke fair hergestellt sind, empfinden viele Jugendliche als zu hoch.

Ob und inwieweit die Jugendlichen sich in Bezug auf Kleidung Maßstäbe des kritischen Konsums zu eigen machen, hängt auch mit der generellen Konsumästhetik in den jeweiligen Lebenswelten zusammen: Materialistische Hedonisten möchten möglichst viel modische Kleidung besitzen. Dabei versuchen sie vor allem Markenartikel zu kaufen, wenn sie dazu finanziell in der Lage sind. Eine besondere Faszination üben auf diese Jugendlichen Luxusmarken aus. Prekäre möchten generell mehr Kleidung kaufen als sie es aktuell tun, bewegen sich aber ständig an den Grenzen ihrer finanziellen Möglichkeiten. Daher ist bei ihnen das Preis-Argument besonders dominant. Expeditiven und Experimentalistischen 
Hedonisten ist ein individueller Kleidungsstil wichtig. Sie möchten tragen, was nicht alle haben. Ihre Kleidung hat den größten Preisrange, da sie teilweise teure Einzelstücke erwerben, die sie mit Kleidung der gängigen Modemarken kombinieren. Second Hand-Läden und der Flohmarkt sind für sie ebenso eine wichtige Quelle. Sozialökologische messen der Kleidung eine weniger hohe Bedeutung bei, für sie ist es nicht wichtig, mit Kleidung etwas darzustellen oder bestimmte Marken haben zu müssen. Konservativ-bürgerliche Jugendliche sehen Kleidung auch nicht als besonders wichtig an und betrachten den Einkauf von Kleidung nicht unbedingt als zentrale Freizeitbeschäftigung. Sie möchten aber immer angemessen gekleidet sein und nicht negativ auffallen. Adaptiv-Pragmatische möchten modisch gekleidet sein und achten darauf, bei den Trends dabei zu sein. Sie tragen das, was "alle anderen auch tragen“. Dies bezieht sich häufig auf bestimmte Farben („,Pastellfarben sind grad in") oder bestimmte Schnitte („Hosen dürfen jetzt wieder einen höheren Bund haben"). Entsprechend ist Kleidung in der Folge-Saison "veraltet" und muss ersetzt werden.

Bei Kleidung sind Preis und Aussehen somit in den meisten Lebenswelten die maßgeblichen Kaufkriterien; die Produktionsbedingungen spielen hingegen (bislang) nur eine untergeordnete Rolle. Das Konsumieren der gängigen günstigen Marken wird an dieser Stelle - vor allem bei Materialistischen Hedonisten, aber auch Adaptiv-Pragmatischen - als alternativlos beschrieben, und wer wenig Geld hat, kann nach Einschätzung der Jugendlichen keine Rücksicht auf die Produktionsbedingungen nehmen, selbst wenn man Kinderarbeit mit aller Entschiedenheit ablehnt.

Am ehesten sind noch die Sozialökologischen und Expeditiven Jugendlichen bereit, beim Kauf von Kleidung bestimmte Marken auszuschließen, allerdings spielen dabei auch ästhetische Präferenzen eine Rolle. Von bestimmten Marken möchte man sich dezidiert abgrenzen. Sehr häufig wird die Marke Primark als Synonym für unfaire Arbeitsbedingungen genannt. Auch H\&M wird als negatives Beispiel hervorgehoben. Viele Jugendliche vermuten allerdings, dass es auch bei den anderen Marken, die von ihnen gekauft werden, kaum besser aussieht. Trotzdem hoffen sie darauf, dass etwas teurere Kleidung auch zu besseren Produktionsbedingungen beiträgt. Sicher sind sie sich aber nicht. 
(1) [INT: IST ES DIR ZUM BEISPIEL WICHTIG, WO DIE KLEIDUNG PRODUZIERT WIRD, DIE DU TRÄGST, UND WER SIE PRODUZIERT HAT?] Nein, eigentlich nicht. [INT: MACHT IHR EUCH DA IM FREUNDESKREIS GEDANKEN?] Nein, halt nur Markensachen, wenn schon. Aber woher man die hat oder wo die produziert werden, interessiert uns gar nicht. [INT: WAS IST DIR DENN WICHTIG, WENN DU KLEIDUNG KAUFST?] Dass sie schön ist und ein bisschen günstig ist. (weiblich, 14 Jahre, Adaptiv-Pragmatische)

(7INT: IST ES FÜR DICH WICHTIG, WO DIE KLEIDUNG PRODUZIERT WIRD, DIE DU TRÄGST, WO DIE GEMACHT WIRD UND UNTER WELCHEN BEDINGUNGEN DER LEBT, DER SIE GEMACHT HAT?] Nein. (weiblich, 17 Jahre, Materialistische Hedonisten)

(8) Es gibt ja Primark, da gehe ich gar nicht rein erst, das finde ich ganz schrecklich. Oder auch wenn meine Freundinnen da waren, dann bin ich nicht sauer auf die, aber dann halte ich denen erst mal so eine Standpauke darüber. H\&M ist wahrscheinlich genauso schlimm, aber ich finde, das ist irgendwie noch mal so ein Unterschied. (weiblich, 17 Jahre, Expeditive)

(7INT: ABER WÜRDEST DU DARAUf SO ACHTEN WOLLEN, ZUM BEISPIEL WO DU DIE SACHEN KAUfST?] Wenn ich das Geld hätte, ja. Das Ding ist ja, wenn man bei H\&M und so kauft, ist das ja genauso. Es ist jetzt nicht so wie früher, wo man gesagt hat, KIK oder was weiß ich nicht was. Diese komischen Billigläden. Jeder lässt ja da produzieren. Auch Nike oder so. Wo will man denn da noch kaufen? Da musst du in irgendwelche Läden gehen, wo dann ein T-Shirt 300 Euro kostet. Und dann sieht es ja nicht mal schön aus. Oder ist halt nicht angesagt oder so. (männlich, 17 Jahre, Adaptiv-Pragmatische)

[INT: AbER WÜRDEST DU ZUM BEISPIEL SAGEN, WENN DU JETZT KEINE NIKES MEHR KAUfEN WÜRDEST, DASS DAS EINFLUSS HABEN KÖNNTE, DASS SICH IRGENDWIE WAS VERBESSERT?] Bestimmt. Also, wenn es viele Leute gibt, die sich dagegen auflehnen, dann verändert es immer was und es muss gar nicht mal sein, dass dann Kinder aufhören zu arbeiten für wenig Geld oder für gar kein Geld, aber einfach in den Köpfen, wenn sich da schon was verändert, ist auch gut. [INT: ABER HÄTTEST DU LUST, DARAUF EINFLUSS ZU NEHMEN?] Ja, aber ich weiß nicht wie. [INT: 
Aber du Willst auch nicht auf deine Nikes verzichten?] Genau. (weiblich, 17 Jahre, Experimentalistische Hedonisten)

[INT: ES GAB JA AUCH SCHON ÖFTER DIE DISKUSSIONEN ÜBER KLEIDUNG, DASS DIE UNTER SCHLECHTEN BEDINGUNGEN HERGESTELLT WIRD UND SOWAS. HAST DU DAVON GEHÖRT?] Ja, habe ich. Also zum Beispiel die Sachen bei Primark, die sind ja sehr billig. Und da haben ja auch Kinder gearbeitet dafür. Und das finde ich auf jeden Fall nicht gut. Also ich schaue jetzt nicht explizit darauf, woher das kommt und ich gucke jetzt genau, von welcher Marke das produziert wird und so weiter. Es ist ja sowieso sehr schwer, was dagegen zu machen, weil das ja sowieso alles in Thailand oder China ist. Aber ich denke, dass irgendwie so Markensachen oder so Sachen, die etwas teurer sind, schon in besseren Verhältnissen produziert wurden. (männlich, 16 Jahre, Materialistische Hedonisten)

( Ich finde das nicht okay, wie die das in Thailand herstellen. Aber ich meine, wie soll man sich denn bitte teure Klamotten kaufen, wenn man dazu nie das Geld hat. Und dann kauft man sich halt doch die anderen. Ich meine, die halten genauso wie die teuren Klamotten, meistens noch länger, habe ich immer das Gefühl. Das ist oft so, dass die billigen einfach mal besser sind als die teuren. Die billigen verfärben sich auch nicht so schnell wie die teuren Klamotten. Und vor allen Dingen kann man das gar nicht mehr garantieren, wo die überhaupt herkommen. (weiblich, 17 Jahre, Konservativ-Bürgerliche)

Also ich probiere es. Ich muss zugeben, dass ich auch manchmal, wenn ich so bei H\&M einkaufen gehe, damit auch Kinderarbeit und sowas unterstütze, was natürlich Kacke ist. Aber da muss ich zugeben, da achte ich dann nicht so sehr drauf. Gerade bei den Klamotten. Auch, wenn es doof ist, aber da muss man ja anfangen zu gucken, wo nicht Kinderarbeit und so etwas mit drin ist. Und das ist super schwer zu finden, weil wirklich fast alles das halt beinhaltet. Und ich habe da auch schon ein paar Sachen drüber gesehen in Studien. Da müsste man auch in irgendwelche Läden gehen, wie heißen die nochmal, so Fair Trade. 
Und das kann ich mir überhaupt nicht leisten, also gar nicht. Das ist die Sache. (männlich, 16 Jahre, Sozialökologische)

\section{Jugendliche finden es schwer, an verlässliche Informationen zu "kritischem Konsum" zu gelangen.}

Kritischer Konsum spielt weder im Schulunterricht noch in Familie oder Freundeskreis eine zentrale Rolle. Nur wenige Jugendliche berichten darüber, dass sie sich gezielt im Internet oder beim Einkaufen über das Thema informieren. Es scheint den Jugendlichen auch (zu) aufwändig und kompliziert, an die notwendigen Informationen zu kommen (z.B. welche Kleidung an welchem Ort unter welchen Bedingungen produziert wurde).

(1) Ich habe mal mir so eine App runtergeladen und dann hatten wir das, glaube ich, auch in der Schule. Da habe ich das gesehen, da gab es so ein Ranking von den ganzen Marken. Und zum Beispiel Marco Polo oder Hilfiger oder so, die waren bei den schlechtesten dabei. Die haben halt schlechte Arbeitsbedingungen, die Arbeiter werden schlecht bezahlt, Kinderarbeit und so was. Und das ist aber bei fast allen so. (weiblich, 17 Jahre, Sozialökologische)

[INT: WÜRDEST DU DICH GERNE MEHR DARÜBER INFORMIEREN ODER MACHST DU DAS SCHON MANCHMAL?] Habe ich noch nicht gemacht, aber ich glaube, das werde ich jetzt echt mal machen, weil ich kann ja im Laden jetzt nicht auf jeden Zettel gucken, wo das herkommt. Das dauert ja eine Ewigkeit. Muss ich dann mal gucken. (männlich, 15 Jahre, Adaptiv-Pragmatische)

Die Jugendlichen bekunden, dass es schwer sei, zwischen richtigen und falschen Informationen zu unterscheiden und äußern nur wenig Vertrauen in Produkt-Siegel. Das Label "Fair Trade" ist zwar bekannt, man ist sich aber unsicher, ob es wirklich verlässlich ist. Fair-Trade-Produkte gelten vor allem auch als zu teuer. Ebenso wird bei "Bio"-Kennzeichnung häufig geurteilt: "Nur weil es drauf steht, muss es nicht drin sein“. Insgesamt ist das Vertrauen in diese Siegel eher gering. 
( Ich kriege immer was von meiner Mutter mit. Dass man gar nicht so vertrauen kann, was geschrieben wird, weil immer wieder was dahinter steckt, was doch falsch ist. Gerade, was so Wolle angeht oder bei den verschiedenen Klamotten oder auch, wie die Umstände von den Näherinnen sind in diesen ganzen Fabriken. (weiblich, 16 Jahre, Expeditive)

( Klar kann man Bio-Fleisch kaufen. Aber in diesen ganzen Enthüllungs-Dokus sieht man ja auch, dass diese Bio-Konzerne auch nur das Gleiche machen. Ich glaube, man muss schon viel recherchieren, um zu wissen, welchen Siegeln man vertrauen kann. (weiblich, 15 Jahre, Expeditive)

(7) [INT: BEI ESSEN, ACHTEST DU DA AUCH DRAUF, WAS DU SO EINKAUFST?] Selten. Bio kann man mittlerweile überall drauf schreiben. Ist das dann wirklich Bio oder schreiben die das da nur so drauf? Aber eigentlich kauf ich nur so die normalen Sachen. (weiblich, 15 Jahre, Sozialökologische)

\section{Kritischer Konsum braucht eine kritische Masse, damit er wirksam ist.}

Die Jugendlichen sind der Meinung, dass sich die globalen Produktionsbedingungen, Tierhaltung und vieles mehr durch kritischen Konsum verändern ließen, sofern - ähnlich wie bei den Themen Umweltschutz und Klimawandel - möglichst viele Konsumentinnen und Konsumenten fair gehandelte und/oder Bio-Produkte kaufen würden. Bislang sieht man aber wenig Sinn darin, als Einzelperson etwas bewegen zu wollen.

Typische

Zitate zur

Illustration
Aber wenn ich jetzt als einziger Mensch keine Klamotten kaufen würde, die mir jetzt gefallen, würde das auch nicht viel weiterhelfen, weil... Wie viele Millionen sind wir jetzt in Deutschland? [INT: 80] 80? Ja, das würde echt nichts ändern so, wenn ich da jetzt ... Wenn da jetzt alle was machen würden, dann würde sich was ändern. So ein bisschen aber nur, weil wir sind ja auch nur ein Land von vielen Ländern. Wir sind 4 Milliarden Menschen auf der Welt? Und die meisten davon sind ja auch Chinesen, glaube ich. [INT: OKAY, ALSO DU SAGST, WENN, DANN 
MÜSSEN ES ALLE MACHEN? ALSO WENN NUR DU WAS MACHST, BRINGT ES GAR NICHTS?] Ja, oder einfach nur Spenden dahin spenden so. Damit sie einfach bessere Bedingungen für die halt aufbauen können. Weil nicht nur die Fabrik sieht ja Kacke aus, sondern einfach so überall, wo die Leute leben, sieht es Kacke aus. Wenn ich jetzt zum Beispiel Videos angeguckt habe über Rio oder so, wie die Leute leben, richtig krass eigentlich. [INT: ALSO DU SAGST, DA MÜSSTE MAN SOZUSAGEN ALLES ÄNDERN, NICHT NUR DIE FABRIK] Ja. (männlich, 15 Jahre, Prekäre)

> Ja natürlich. Also ich glaube, wenn jetzt weniger Menschen, keine Ahnung, Billig-Kleidung irgendwie aus diesen Ein-Euro-Läden kaufen würden, würde das auf jeden Fall mehr bringen oder halt so, keine Ahnung, keine tierischen Produkte aus der Massentierhaltung und sowas, wenn man sich halt dafür wirklich einsetzen würde, nicht das kaufen würde, würde es echt viel ausmachen, klar. (weiblich, 17 Jahre, Adaptiv-Pragmatische)

I Ich glaube aber, wenn eine Menschenmasse daran denken würde, Sachen woanders zu kaufen, Fair-Tade oder so, dann würde das natürlich was ändern. Aber wenn jetzt eine Person persönlich sehr genau darauf achtet, was sie kauft, ändert das glaube ich nicht viel. (weiblich, 15 Jahre, Experimentalistische Hedonisten)

(7) Das müsste wenn dann eine Massenregung sein. [INT: GLAUBST DU, MAN KÖNNTE DAS IRGENDWIE BEEINFLUSSEN, WENN DU DA NICHT KAUFEN WÜRDEST? GLAUBST DU, DAS HÄTTE EINEN POSITIVEN EFFEKT?] Na ja, ich denke, dass das etwas bringt, weil wenn jeder sich irgendwie denkt, es bringt nichts, dann macht es ja auch niemand. Ja, ich denke schon, dass das etwas bringt. Man muss halt nur auch andere davon überzeugen. Jeder muss da halt ein bisschen so denken, dass man das in die Hand nehmen kann. (weiblich, 15 Jahre, Adaptiv-Pragmatische)

[INT: GLAUBSt Du, DASS UNSERE KONSUMENTSCHEIDUNGEN EINFLUSS HABEN?] Auf dem Weltmarkt? Es ist immer schwer, das wirklich zu glauben. Man sagt ja immer, dass auch der Einzelne das Ganze mit beeinflussen kann. Aber es gibt so viele andere, die nicht daran denken. Und deswegen ist es immer etwas schwer, sich das bewusst zu machen. 
Man macht es ja nur als Einzelner. Und alles andere passiert ja trotzdem. (weiblich, 17 Jahre, Adaptiv-Pragmatische)

[INT: GLAUBST DU, DASS WIR HIER IRGENDETWAS BEEINFLUSSEN KÖNNEN?] Ja, wenn man das nicht mehr kaufen würde. Aber ich glaube, es ist total schwer, in der Massenproduktion alle Menschen umzustimmen, damit sie das nicht mehr kaufen. Und irgendwann würde es auch einfach verheimlicht, dass Kinderarbeit gemacht wird. [INT: WÄRE ES INTERESSANT FÜR DICH, AUF IRGENDEINE WEISE DARAUF EINFLUSS ZU NEHMEN?] ICh glaube, das wäre total interessant. Aber es wäre schwer, das zu machen. Es wird kaum jemand auf einen hören. (weiblich, 14 Jahre, Adaptiv-Pragmatische)

\section{Jugendliche zweifeln daran, dass sich durch kritischen Konsum die Verhältnisse wirklich ändern können.}

Verändertes Konsumverhalten ist aus Sicht der Jugendlichen kein Allheilmittel. Beispielsweise ließe sich Kinderarbeit weder global verhindern, noch gegenüber den Kunden verheimlichen. Es wäre auch nicht wünschenswert, wenn die Menschen an den Produktionsorten gar keine Arbeit und kein Einkommen mehr hätten, weil ihre Produkte nicht mehr gekauft würden. Auch hinsichtlich des Lebensmittelkonsums vermuten einzelne Jugendliche Probleme. Denn selbst wenn sich alle Menschen vegetarisch oder vegan ernähren würden, änderte das nach ihrer Auffassung nichts an der grundsätzlichen Problematik, dass insgesamt zu viele Menschen ernährt werden müssten.

Typische

Zitate zur

Illustration
Also generell mit dem Thema Kinderarbeit, natürlich sollte man da was ändern. Aber es kann halt nicht viel geholfen werden. Und ich glaube, die Leute, die da den Job haben, wenn man ihnen den wegnehmen würde und die dann halt wieder gar nichts hätten, wäre das natürlich auch schlecht. Weil sie dann überhaupt gar kein Geld verdienen und kein Essen haben und dann letztendlich komplett verhungern. Also für mich wäre es schon okay, wenn Erwachsene arbeiten. Natürlich unter nicht so schlechten Bedingungen und mit besserem Lohn, aber ich 
glaube, es wäre schon schlechter, wenn man ihnen einfach so die Arbeit wegnimmt. Sie verdienen dann überhaupt kein Geld mehr, haben kein Essen mehr und können ihre Familie nicht mehr ernähren. (weiblich, 15 Jahre, Experimentalistische Hedonisten)

Ich weiß, dass bei Primark zum Beispiel Kinderarbeit ganz viel drin steckt, und ich finde es schade. Andererseits denke ich mir, wenn ich jetzt aufhöre damit, dann bringt es nichts, weil diese Leute, die das da haben oder die da kaufen, weil ich jetzt aufhöre, hören die nicht auf. Also es wird immer irgendwo Kinderarbeit geben, glaube ich. Das kann man nicht unterbinden, das geht nicht. (weiblich, 17 Jahre, Materialistische Hedonisten)

( Ich weiß nicht, ob es Vegetarier oder Veganer richtig machen. Klar, ich kann das nachvollziehen. Ich bin selber kein Vegetarier oder Veganer. Aber wenn plötzlich alle Fleischesser nur noch Soja essen würden, gäbe es damit sicherlich auch Probleme, wenn nur noch überall Soja angebaut werden würde. Ich weiß nicht, ob das die Lösung ist. Ich denke, das Problem ist, dass es so viele Menschen gibt. Und diese vielen Menschen wollen ja alle auch etwas essen. Und das Essen muss man irgendwoher bekommen. Bei Kartoffeln ist es sicher egal, wo sie gezüchtet werden. Aber bei lebenden Tieren eben nicht. Das eigentliche Problem ist, dass es so unglaublich viele Menschen auf der Welt gibt. (weiblich, 15 Jahre, Expeditive)

Open Access Dieses Kapitel wird unter der Creative Commons Namensnennung - Nicht kommerziell 2.5 International Lizenz (http://creativecommons.org/licenses/by-nc/2.5/deed.de) veröffentlicht, welche für nicht kommerzielle Zwecke die Nutzung, Verbreitung und Wiedergabe in jeglichem Medium und Format erlaubt, sofern Sie den/die ursprünglichen Autor(en), den Titel des Werks und die Quelle ordnungsgemäß nennen, einen Link zur Creative Commons Lizenz beifügen und im Falle einer Abwandlung durch einen entsprechenden Hinweis deutlich erkennbar machen, dass Änderungen vorgenommen wurden.

Die in diesem Kapitel enthaltenen Bilder und sonstiges Drittmaterial unterliegen ebenfalls der genannten Creative Commons Lizenz, sofern sich aus der Abbildungslegende nichts anderes ergibt. Sofern das betreffende Material nicht unter der genannten Creative Commons Lizenz steht und die betreffende Handlung nicht nach gesetzlichen Vorschriften erlaubt ist, ist auch für die oben aufgeführten nicht-kommerziellen Weiterverwendungen des Materials die Einwilligung des jeweiligen Rechteinhabers einzuholen. 